\title{
Directional Fluorescence Spectral Narrowing in All-Polymer Microcavities Doped with CdSe/CdS Dot-in-Rod Nanocrystals
}

\author{
Giovanni Manfredi, $^{\dagger}$ Paola Lova, ${ }^{\dagger}$ Francesco Di Stasio, $^{\ddagger}{ }^{\S}$ Roman Krahne, $^{\ddagger \oplus}$ and Davide Comoretto ${ }^{*}, \dagger(0)$ \\ ${ }^{\dagger}$ Dipartimento di Chimica e Chimica Industriale, Università di Genova, Genova, Italy \\ ${ }^{\ddagger}$ Nanochemistry Department, Istituto Italiano di Tecnologia, Genova, Italy
}

Supporting Information

ABSTRACT: We report on the fluorescence properties of high optical quality all-polymer planar microcavities embedding core-shell dot-in-rod CdSe/CdS nanocrystals. Properly tuned microcavities allow a 10 -fold sharpening of the nanocrystals fluorescence spectrum, resulting in a reduction of the bandwidth from 24 to $2.4 \mathrm{~nm}$, which corresponds to a quality factor larger than 250. A 5-fold peak photoluminescence intensity enhancement is measured, while the overall number of emitted photons is reduced. Time-resolved photoluminescence and quantum yield for microcavities and suitable references show the presence of two decays related to differences in nanocrystal size distribution. The slower decay rate, which becomes

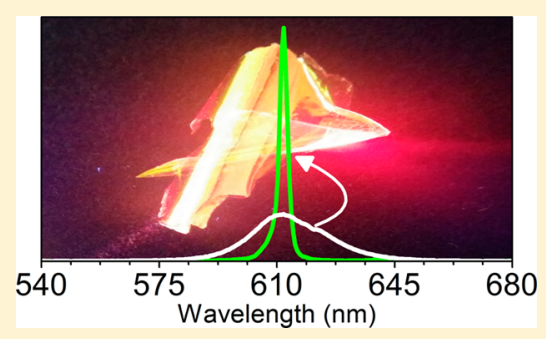
faster when the nanocrystals are embedded into the microcavity, is assigned to longer nanorods with emission spectrally overlapped to the cavity mode. Conversely, the short-living component, which is assigned to an impurity of shorter nanorods, remains unaffected by the microcavity.

KEYWORDS: polymer, microcavity, nanocrystals, quantum dots, photonic crystals

olloidal nanocrystals have attracted considerable attention in the field of light emitting devices thanks to their high fluorescence quantum yield (QY) and spectral tunability via electronic structure engineering and surface functionalization. Indeed, their composition and shape can be adjusted to obtain materials with tailored and stable electronic properties. ${ }^{1}$ Nanocrystals of different shapes such as CdSe nanoplatelets $^{2}$ and core-shell architectures as CdSe/CdS dot-inrods $^{3}$ or giant-shells $s^{4,5}$ have been used in several lightemitting applications, ${ }^{6-10}$ including lasers working both in the pulsed and in the continuous wave regime. ${ }^{11-17}$ To this end, a careful surface functionalization improves passivation, thus, reducing photoluminescence (PL) quenching defects, ${ }^{18-22}$ while allowing solubility in many organic and polar solvents, including water, ${ }^{23-27}$ and promoting self-organization and preparation of nanocomposites for solid state photonic structures and devices. ${ }^{28-36}$ Among these structures, photonic crystals, which are dielectric lattices where materials with different refractive indices are periodically alternated at the submicrometric scale, have been demonstrated effective for light propagation control and light-matter interaction management. ${ }^{37,38}$ While inorganic photonic crystals are widely used, their organic counterparts recently gained growing interest owing to their simple preparation methods, low weight, and extraordinary mechanical properties. ${ }^{30}$ In particular, polymer planar photonic crystals such as Distributed Bragg Reflectors (DBRs) and microcavities are promising for mass production thanks to established fabrication technologies currently used in packaging industry. ${ }^{39-44}$ On the lab scale, these multilayered structures can be successfully prepared by spin-coating of polymers or inorganic nanoparticles, and by block copolymer self-assembly. ${ }^{45,46}$ While the latter needs complicated and expensive synthetic routes, ${ }^{47,48}$ spin-coating inorganic nanoparticles multilayers requires time-consuming postdeposition annealing. ${ }^{49-53}$ Spin-coating of polymer solutions is instead a fast and low cost alternative, that provides high optical quality DBRs and microcavities. Moreover, such structures can be peeled-off from the substrate leading to free-standing and flexible photonic crystals adaptable to preformed surfaces (Figure 1a)..$^{29,41,42,54-65}$ In the framework of organic photonics, all-polymer DBRs and microcavities have been successfully demonstrated as efficient sensors, ${ }^{64,66,67}$ lasers, ${ }^{28,56,57,68}$ and all-optical modulators. ${ }^{69-71}$ Concerning optical resonators, so far the research activities have been focused on lasing properties such as pump threshold and emission narrowing, while material issues related to the optical quality of polymer planar microcavities, their spectroscopic properties, field confinement, and directional emission have not been exhaustively addressed yet. $^{28,56,57,68}$

In this work, we investigate high quality, large area, all-polymer planar microcavities embedding $\mathrm{CdSe} / \mathrm{CdS}$ dot-in-rods (DiRs) in the cavity layer (Figure $1 \mathrm{~b}$ ). We report on the dual emission of DiRs:polystyrene (PS) nanocomposite and on line-narrowing and directional redistribution of the fluorescence oscillator strength in all-polymer microcavities. Moreover, we discuss nanosecond photoluminescence decay dynamics and quantum yields showing the effect of field confinement on the microcavity photophysics.

Received: March 30, 2017

Published: June 8, 2017 


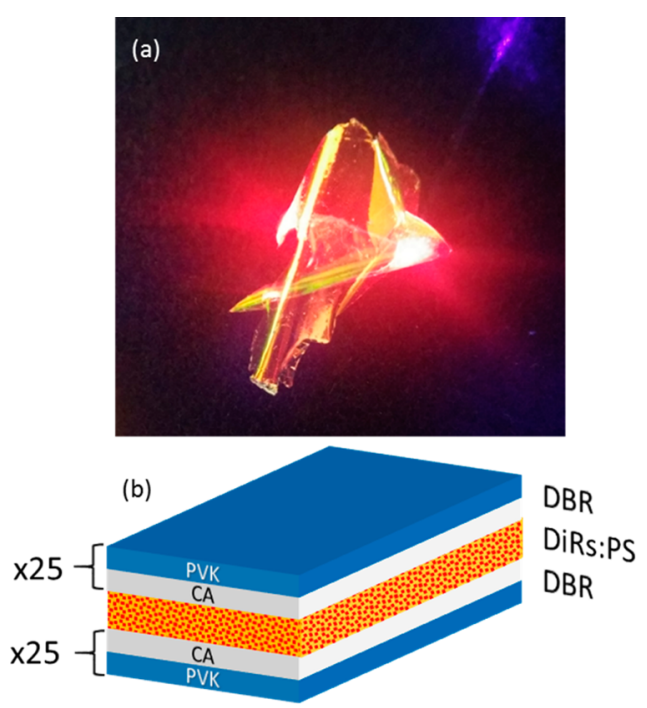

Figure 1. (a) Freestanding rolled-up microcavity under violet laser excitation where the bright DiRs red fluorescence is observed. (b) Scheme of the planar microcavity.

\section{RESULTS AND DISCUSSION}

In this work, we focus on CdSe/CdS DiRs with a diameter of $4.7 \mathrm{~nm}$ and a typical length of $\sim 35 \mathrm{~nm}$; however, displays some dispersion in length together with a second population of much shorter impuritiess, as retrieved from the Transmission Electron Microscopy (TEM) micrographs reported in Supporting Information, Figure S1. Figure 2a shows absorbance and PL spectra of DiRs dispersed in toluene. The well-defined absorbance peaks positioned at 602 and $557 \mathrm{~nm}$ are related to

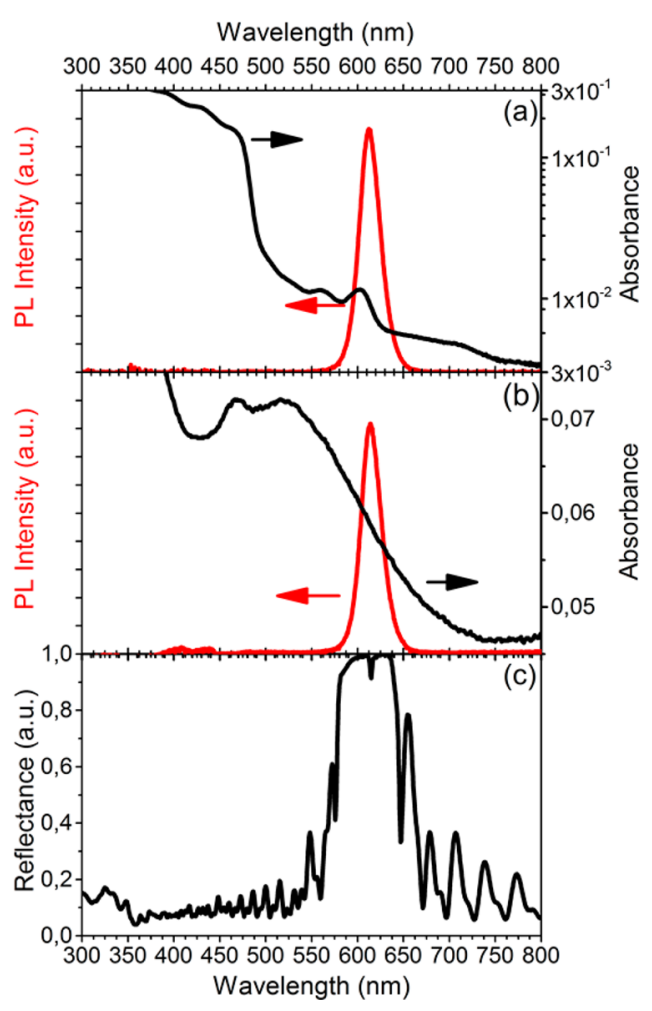

Figure 2. Absorption (black) and fluorescence (red) spectra of (a) nanocrystals dispersed in toluene and (b) nanocrystals dispersed in PS. (c) Normalized reflectance spectrum of the microcavity. the CdSe core. ${ }^{3}$ The strong absorption onset around $500 \mathrm{~nm}$ is assigned to the bulk-like rod-shaped CdS shell. The remarkable difference in intensity comparing CdSe to $\mathrm{CdS}$ features is due to the larger amount of CdS in the nanocrystals. DiRs show a slightly asymmetric PL spectrum centered at $612 \mathrm{~nm}$ with $24 \mathrm{~nm}(79 \mathrm{meV})$ full width at half-maximum (fwhm). A $10 \mathrm{~nm}$ Stokes shift with respect to the CdSe core band edge is detected.

To embed DiRs in high optical quality all-polymer microcavities, a suitable nanocomposite has been prepared. For this purpose, DiRs were dispersed into a polystyrene matrix (DiRs:PS), as described in the Methods section. Figure $2 \mathrm{~b}$ reports the absorption and fluorescence spectra of the DiRs:PS nanocomposite spin-cast films, that show remarkable differences in absorption from the toluene DiRs dispersion (Figure 2a). The features in the range between 500 and $650 \mathrm{~nm}$, which were originally observed in the suspension, appear masked by a broad tail assigned to light scattering generated by the large amount of DiRs loaded into the nanocomposite (see also Supporting Information, Figure S2). The CdS shell absorption is still detected at $480 \mathrm{~nm}$ as a peak. Interestingly, even with the different absorption profiles, the PL line shape in the composite film remains unchanged with respect to the toluene solution, suggesting that light scattering induced by heavily loaded DiRs does not affect the electronic properties of the emitters, which retain their nanocrystal identity. Indeed, DiRs coalescence can be excluded since confocal reflection and fluorescence microscopy images (Supporting Information, Figure S2) reveal a homogeneous high density distribution of small, submicrometric emitting spots instead of a uniform fluorescent film.

A careful analysis of the PL spectra shows the effect of the DiRs nonmonodisperse size distribution previously observed in the TEM images (Supporting Information, Figure S1). Indeed, the PL spectra can be deconvolved into two Voigt shaped components centered at 608 (peak 1) and $617 \mathrm{~nm}$ (peak 2; see Supporting Information, Figure S3 and Table S1). The Voigt line shape accounts for inhomogeneous broadening in the system. The same deconvolution with very similar parameters also holds for DiRs dispersed in toluene. According to the reported size distribution (Supporting Information, Figure S1) and to the known relation between PL and quantum dots size, we assign the low energy peak $(617 \mathrm{~nm})$ to longer DiRs (with average length of $35 \mathrm{~nm}$ ), while the high energy one $(608 \mathrm{~nm})$ is assigned to the much shorter DiRs. ${ }^{1,3}$

A nanocomposite thin film identical to the one used to record the spectra of Figure $2 \mathrm{~b}$ has been embedded between two identical $\lambda / 4$ DBRs made of 25 spin-cast bilayers of poly(N-vinylcarbazole) (PVK) and cellulose acetate (CA). The microcavity reflectance spectrum shows an intense and broad peak between 580 and $646 \mathrm{~nm}$ assigned to the photonic band gap (PBG, Figure 2c). The wide fwhm (66 nm) arises from the relatively large (compared to other polymer systems) dielectric contrast between PVK $(n \sim 1.66$ at $\lambda=600 \mathrm{~nm})$ and $\mathrm{CA}(n \sim 1.48$ at $\lambda=800 \mathrm{~nm}) .^{72}$ Within the PBG, we observe a sharp minimum at $613 \mathrm{~nm}$ assigned to the cavity mode, while the background of the reflectance spectrum is modulated by interference fringes. The absorption of the PVK aromatic rings $^{73-75}$ affects the reflectance spectrum below $350 \mathrm{~nm}$ and overlaps the CdS shell absorption. The second order PBG, which is expected at $\sim 300 \mathrm{~nm}$, is not observed, confirming that the thickness of the layers in the dielectric mirrors fulfill the $\lambda / 4$ condition. We remind that under such conditions, the bandwidth of the PBG can be analytically calculated as 
$\Delta E=\frac{4}{\pi} E \frac{\left|n_{h}-n_{l}\right|}{n_{h}+n_{l}} \cdot{ }^{30}$ According to this equation and to refractive index dispersions previously reported, ${ }^{72}$ the expected bandwidth for this system is $60 \mathrm{~nm}$, which is in good agreement with the experimental value $(66 \mathrm{~nm})$.

Figure 3a compares the transmittance spectrum of the microcavity to the one calculated via the transfer matrix
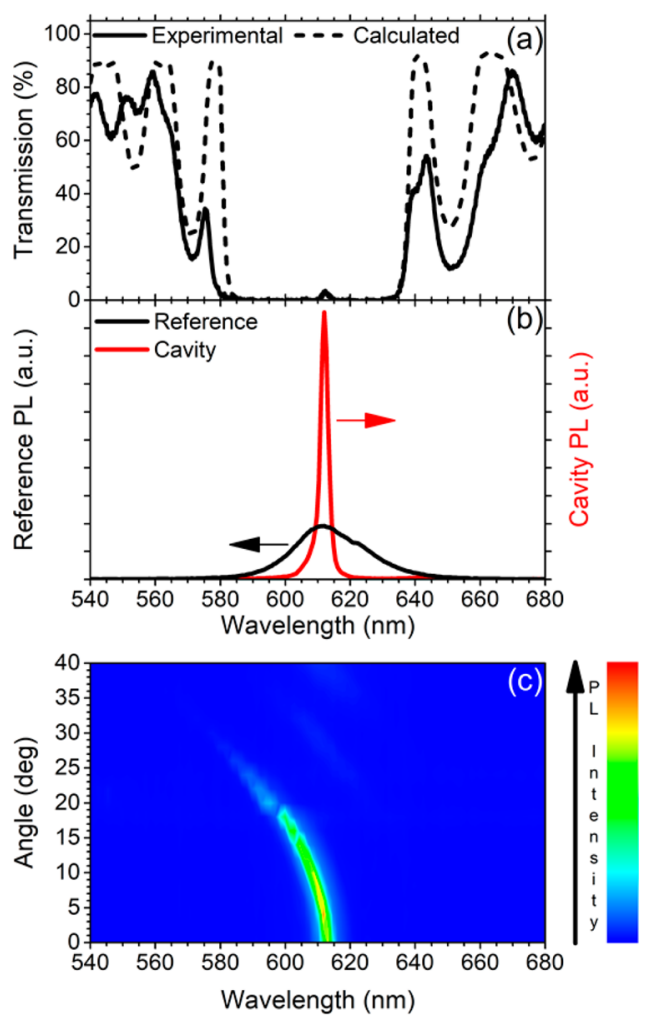

Figure 3. (a) Experimental (continuous line) and calculated (dashed line) microcavity transmission spectra. (b) PL spectra for a microcavity (in red) and for a reference sample (in black) recorded in the very same conditions. (c) Contour plot of the microcavity fluorescence spectra as a function of the collection angle.

method $(\mathrm{TMM})^{76}$ using the refractive index dispersions of PVK and CA reported in literature ${ }^{72}$ and preliminary complex optical functions for DiRs:PS (see Supporting Information, Figure S4). The simulation has been used to fit the thickness of the layers composing the microcavity and the results are $94 \mathrm{~nm}$ for PVK, $100 \mathrm{~nm}$ for CA, and $208 \mathrm{~nm}$ for DiRs:PS nanocomposite. These values are in good agreement with those reported in our previous works ${ }^{69,77,78}$ and with those calculated by the analytical formulas for the $\lambda / 4$ condition (92 nm for PVK and $104 \mathrm{~nm}$ for CA). ${ }^{30}$ In addition, the calculated thickness of the nanocomposite layer successfully matches the $\lambda / 2$ condition $^{30,79}(193 \mathrm{~nm})$. Transmittance spectrum shows a broad band with negligible values in the range 580-646 nm containing a sharp and weak maximum centered at $613 \mathrm{~nm}$. These two features correspond to the microcavity PBG and cavity mode, respectively. The calculated spectrum reproduces all the major features of the experimental one (PBG width, cavity mode position, interference fringes). Moreover, the simulation perfectly accounts for polarized angle-resolved transmittance spectra (Supporting Information, Figure S5), further demonstrating the excellent optical quality of our all-polymer microcavities. ${ }^{30,80}$
Figure $3 \mathrm{~b}$ highlights the spectral reshaping induced by the microcavity on the PL of DiRs:PS nanocomposite with respect to the reference film recorded under the very same experimental conditions. The modified photonic environment induces a factor 10 sharpening of the PL spectrum, whose fwhm changes from $24 \mathrm{~nm}$ for the reference to $2.4 \mathrm{~nm}$ for the microcavity (see also Supporting Information, Figure S6, for a comparison of peak normalized PL spectra). In addition, for the microcavity, PL peak intensity is $\sim 5 \times$ larger than for the reference. The microcavity quality factor $(Q=\lambda / \Delta \lambda=255)$ is the largest so far reported for planar all-polymer microcavities. $^{28,41,56,57,77,80}$ The PL spectrum of the nanocomposite has been dramatically reshaped with a strong reduction of the intensity at the PBG spectral region, and a strong increase at the cavity mode. This spectral redistribution of the PL oscillator strength agrees with previous findings obtained on different emitting systems having much broader PL spectrum ${ }^{77,80}$ and indicates that a very fine-tuning can be easily obtained with spun-cast all-polymer large area microcavities.

The spectral redistribution of PL can be explained using the modified Fermi's Golden Rule within the photonic crystal. The traditional formula for the transition rate within the timedependent weak perturbation approach:

$$
W(\omega)=\frac{2 \pi}{\hbar}|\langle\boldsymbol{\mu} \cdot \mathbf{E}\rangle|^{2} \rho(\omega)
$$

is modified by the photonic crystal. The electric field $\mathbf{E}$ in a photonic crystal is related to the allowed optical modes $\mathbf{E}_{n, k}$ $(\mathbf{r}, \omega)$ within the dielectric lattice. The density of states $\rho(\omega)$ must be replaced by the local density of photonic states $\left(\rho_{1}(\mathbf{r}, \omega)\right):^{80-86}$

$$
\rho_{l}(\mathbf{r}, \omega)=\frac{1}{(2 \pi)^{3}} \sum_{n} \int d^{3} k\left|\boldsymbol{\mu} \cdot \mathbf{E}_{n, k}(r)\right|^{2} \delta\left(\omega-\omega_{n, k}\right)
$$

leading to

$$
W(\omega)=\frac{2 \pi}{\hbar} \rho_{l}(\mathbf{r}, \omega)
$$

In eq $2, \boldsymbol{\mu}$ is the matrix element of the dipole moment between the final |f $\rangle$ and initial $|i\rangle$ electronic states. In the case of an emitter embedded into a homogeneous isotropic medium, the density of photonic states shows an $\omega^{2}$ dependence, while in a microcavity its spectral shape becomes a crucial factor (eq 2). ${ }^{81}$ At the PBG energies, $\rho_{1}(\mathbf{r}, \omega)$ is negligible, while at the cavity mode, it strongly grows in a sharp frequency interval, thus, enhancing $W(\omega)$. The width of the enhanced region is related to the cavity volume, which depends on the dielectric contrast between the materials used for the DBR mirrors. ${ }^{30,80}$ Therefore, the origin of the observed PL spectral redistribution so far discussed (Figure $3 \mathrm{~b}$ ) is the spectral shape of $\rho_{1}(\mathbf{r}, \omega)$.

An additional consequence of the eqs 2 and 3 concerns the directionality of the emission. Figure $3 c$ shows the steady-state PL spectra of the microcavity collected at angles between 0 and $32^{\circ}$. The cavity emission shifts toward higher energy increasing the detection angle, according to the PBG dispersion previously described (see Supporting Information, Figure S5, for the transmittance spectrum of the system and Supporting Information, Figure S7, for the PL spectra as a function of wavelength and angle of collection). Increasing the detection angle up to $15^{\circ}$, the $\mathrm{PL}$ intensity remains almost constant. Conversely, over $15^{\circ}$, the cavity mode does not efficiently overlap the PL of the DiRs:PS nanocomposites (Figure 2b), and the signal starts 
Table 1. Lifetime $\left(\tau_{j}\right)$ and Relative Intensity $\left(A_{j}\right)$ of the Different Decays as Deduced from the Fitting Procedure

\begin{tabular}{lcccccc} 
& $\tau_{1}(\mathrm{~ns})$ & $A_{1}$ & $\tau_{2}(\mathrm{~ns})$ & $A_{2}$ & $\tau_{3}(\mathrm{~ns})$ & \multicolumn{2}{c}{$A_{3}$} \\
microcavity $(613 \mathrm{~nm})$ & $6.7 \pm 0.4$ & $0.13 \pm 0.04$ & $12.0 \pm 0.1$ & $0.83 \pm 0.04$ & $40.3 \pm 0.8$ \\
reference $(613 \mathrm{~nm})$ & $6.4 \pm 0.7$ & $0.35 \pm 0.05$ & $13.2 \pm 0.3$ & $0.61 \pm 0.06$ & $43 \pm 4$ \\
reference $(600 \mathrm{~nm})$ & $5.2 \pm 0.5$ & $0.56 \pm 0.06$ & $13.1 \pm 1$ & $0.42 \pm 0.07$ & $56 \pm 5$ & $0.04 \pm 0.01$ \\
reference $(630 \mathrm{~nm})$ & & 0 & $12.6 \pm 0.6$ & $0.86 \pm 0.02$ & $51 \pm 3$ & 0.02 \\
\end{tabular}

fading. Indeed, Figure $3 \mathrm{c}$ demonstrates that, in addition to the spectral redistribution, a dramatic change in the spatial DiRs emission occurs from the almost lambertian character of the reference to a highly directional fluorescence given by the microcavity, which allows light propagation only along its axis. $^{84}$

Despite the observed peak enhancement, the microcavity PL intensity integrated over the full spectrum is reduced by 25\% compared to the reference one, indicating that the overall emission is not clearly enhanced. ${ }^{84}$ Indeed, PL QY measurements (Table 1) show a reduction of PL efficiency in the microcavity $(6.5 \%)$ with respect to the reference (33\%). This result can be mainly explained by self-absorption caused by the reduced DiRs:PS nanocomposite Stokes shift $(\sim 10 \mathrm{~nm})$. In spite of the weak CdSe core absorption, the self-absorption is intensified by the very long photon dwelling time inside the cavity. Indeed, while the photon transit time through a reference film having the very same thickness as the cavity can be easily calculated as $\sim 1$ fs $\left(n_{\text {cavity }} d_{\text {cavity }} / c=1.6 \times 193 \mathrm{~nm} / c\right.$, $c$ speed of light), the dwelling time inside the cavity is instead deeply affected by the presence of the DBR mirrors: photons are confined by reflections inside the cavity layer, and their dwelling time is dependent on the quality factor of the structure $\left(\tau_{\mathrm{d}}=Q /(2 \pi \omega)=83 \mathrm{fs}\right)$. In this way, the probability of selfabsorption is greatly increased when compared to the isolated reference film. The fate of each self-absorbed fluorescence photons is now to be either re-emitted or to decay through nonradiative processes. In this way, a significant reduction of the microcavity QY is induced as experimentally observed. Notice that, being the DiRs:PS nanocomposites capped by CA layers both in the reference and in the microcavity (see Methods for details) we do not need to claim for additional nonradiative recombination pathways to explain the QY reduction observed for the microcavity.

Additional insights on this topic can be obtained by the analysis of the nanosecond transient PL decays. According to eq 3 , the local density of photonic states influences the radiative lifetime, which is related to the transition rate by

$$
\tau_{\mathrm{r}}^{-1}=W_{\mathrm{r}}(\omega)
$$

and, therefore, to the net radiative rate enhancement. According to eqs 3 and 4, a reduction of PL lifetime should be observed for the microcavity with respect to the reference DiRs:PS nanocomposite film. The modifications described by eqs 3 and 4 should reduce emission lifetime. To investigate this effect, we measured the PL decays for the composite DiRs:PS reference film and for the microcavity at the cavity mode wavelength in the spectral range 610.5-615.5 nm (Figure 4). Compared to the reference, the microcavity PL shows a slower decay for the initial 50 ns. For longer delays the two decays are instead similar. This behavior is clearly in contrast with the reduction of lifetime expected from eqs 3 and 4 considering the effect of radiative decays only.

To better understand this puzzling behavior, we fitted the decays by triexponential functions. We are aware that different
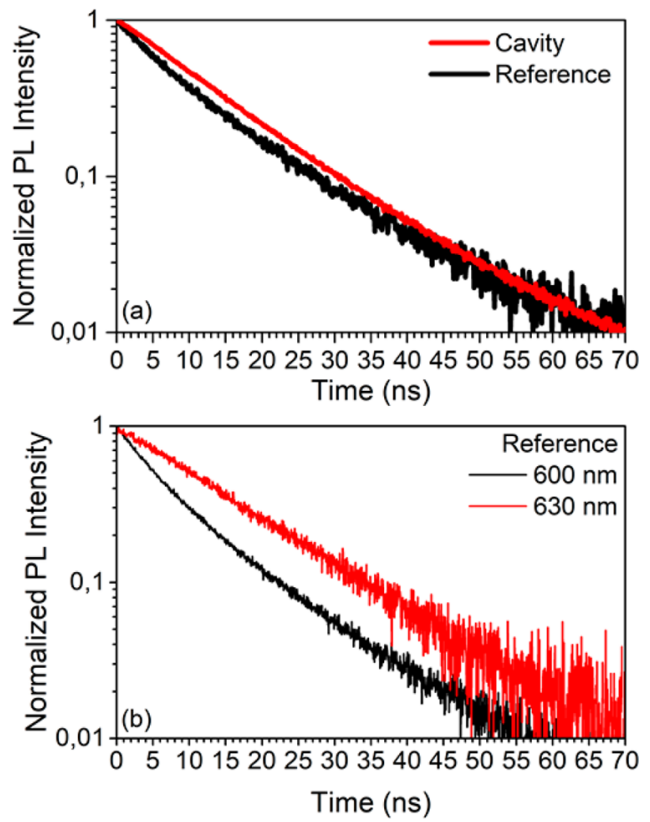

Figure 4. (a) Normalized DIRs PL decay for microcavity (red line) and reference (black line). (b) Spectrally resolved decay for the reference: $600 \mathrm{~nm}$, mainly peak 1 component; $630 \mathrm{~nm}$, mainly peak 2 component.

functional decays have been used in literature to describe the photophysics of single nanocrystals or highly controlled suspensions, ${ }^{87-91}$ but we feel safe to start the discussion on our complex solid state samples (containing both DiRs and impurities) with the simpler multiexponential kinetics. Table 1 summarizes both lifetimes $\left(\tau_{i}\right)$ and relative weights $\left(A_{i}\right)$. Notice that the decay of DiRs in toluene suspension can be successfully fitted by a triexponential function, too (Supporting Information, Figure S8 and Table S2).

For all the decays, the long-living PL component ( $40 \mathrm{~ns}$ ) accounts only for a few percent of the total emission. According to literature, this very weak contribution can be assigned to surface traps and will not be further discussed. ${ }^{92,93}$ Since this contribution accounts only for few percent to the overall microcavity PL kinetics, we discard it from further discussions.

Our attention will be instead focused on the two faster contributes that we assign to the different emissions due to the DiRs size distribution (Supporting Information, Figures S1b and S3). This assignment is supported by the strong spectral dependence of the decays. At $600 \mathrm{~nm}$, where the small impurities contribution (peak 1) to the overall PL is strong (see Figure S3b for details), the fast decay (5.2 ns) contributes $56 \%$ to the decay (see Table 1). While the slower decay ( $13.1 \mathrm{~ns}$ ) has a $42 \%$ weight only. Very different is instead the decay at $630 \mathrm{~nm}$ where the contribution of long DiRs (peak 2) is dominant (see again Figure S3b for details). There, the fast component disappears while the slower one (12.6 ns) grows to $86 \%$ of the overall decay. 
Let us now focus on the PL decay at the cavity mode wavelength $(613 \mathrm{~nm})$. We observe that the relative weights of the fast and slow PL decay components $\left(A_{1}\right.$ and $\left.A_{2}\right)$ are $35 \%$ and $61 \%$, respectively. Analyzing the deconvoluted DiRs PL spectra (Supporting Information, Figure S3a) in the range $610.5-615.5 \mathrm{~nm}$ (the cavity mode spectral region), we observe that the weight of the two contribution is very similar $(\sim 35 \%$ for the short wavelength emission (peak 1) and $\sim 65 \%$ for the long wavelength one (peak 2); see Supporting Information, Figure S3 and Table S1). Considering these findings, we assign the fast decay $\tau_{1}$ to the short wavelength emission $(608 \mathrm{~nm})$, which generates form the short DiRs population, while $\tau_{2}$ is assigned to the long wavelength PL component (613 nm) related to DiRs with $\sim 35 \mathrm{~nm}$ length. In agreement with this assignment, longer DiRs, which have a better surface passivation due to the more developed $\mathrm{CdS}$ shell, are also the ones showing reduced nonradiative recombination rate, hence, longer lifetimes. ${ }^{19-21}$ Unfortunately, a direct correlation between the radiative and nonradiative rates through the overall quantum yield (see Table 1) is not straightforward when multiple decays simultaneously occurs, since the absorption and QY for the single DiRs populations cannot be measured.

We discuss now the role of the microcavity on the fluorescence decay. When embedded in the microcavity, the DiRs show a smaller $\tau_{2}$ than in the reference sample, whereas $\tau_{1}$ remains constant within the experimental uncertainty. The relative weight $A_{2}$, instead, drastically grows inside the microcavity (from 61 to $83 \%$, see Table 1 ), while $A_{1}$ decreases (from 35 to $13 \%$, see Table 1). The different response is related to the unbalanced spectral overlap between the cavity mode $(613 \mathrm{~nm})$ and the two different emissions. Indeed, an excellent overlap is found for the slow decay (PL centered at $617 \mathrm{~nm}$ ) while it is reduced for the fast component (PL peaked at $608 \mathrm{~nm}$ ). Then, an effective enhancement of PL peak intensity (almost 10X) and lifetime reduction (from 13.2 to $12 \mathrm{~ns}$ ) is observed for the slower component. On the other hand, for the fast decay $(\sim 6 \mathrm{~ns})$, the poor spectral overlap does not allow a significant change in the emission rate, hence the component retains the same lifetime. These changes might suggest the presence of Purcell effect which is however limited to the PL contribution of the DiRs longer in size (peak 2). We are aware that the decrease in $\tau_{2}$ is not sufficient to definitely prove the Purcell effect, which is indeed very difficult to observe. ${ }^{94,95}$ PL lifetime reduction might be caused not only by the photonic cavity environment, but also by an increase of the nonradiative decays rate introduced by microcavity growth. However, as previously discussed for the QY, we do not expect any change in the nonradiative recombination rate for DiR:PS nanocomposites being capped and prepared in the very same way both for the reference and for the microcavity. For all these reasons, we are left to assume that changes might occur only in the radiative rates of the long size DiRs. Additional work will be necessary to further support this interpretation. To this end, a very important parameter is the dielectric contrast of the polymers used to grow the DBR mirrors, which currently limits the cavity volume. ${ }^{28,84,86,94,95}$ In this work, we used commercial polymers having the largest dielectric contrast available over a broad spectral range, which however does not seem enough to massively increase the radiative rate. Novel polymer architectures like hyperbranched polyvinyl sulfides ${ }^{77}$ or heavily doped nanocomposites with high refractive index guest medium ${ }^{64,96,97}$ could be a solutions to substantially increase the dielectric contrast and then significantly reduce the cavity mode volume, which is one of the main parameters governing the lightmatter interaction in confined systems and then enhance phenomena like the Purcell effect.

\section{CONCLUSIONS}

We reported solution-based bottom-up fabrication of all-polymer planar microcavities embedding CdSe/CdS DiRs nanocrystals as fluorescent medium. DiRs are dispersed in a polystyrene matrix to obtain a highly process able nanocomposite. Optical investigation of the microcavity shows a 10-fold sharpening and a five-time increase of the emission at the cavity mode wavelength, while the overall number of emitted photons is reduced. Our microcavity shows a $Q$-factor of 255 that, to the best of our knowledge, is the highest $Q$-factor reported so far for all-polymer planar microcavities. PL spectra can be deconvolved into two components related to long DiRs and small impurities. For the high-energy and fast component due to short impurities (peak 1), weakly resonant with the cavity mode, no changes in lifetime are observed. For the resonant longer wavelength emission due to long DiRs (peak 2), a 10\% lifetime reduction is observed that might result from Purcell effect.

\section{METHODS}

The DiRs used in this work were synthesized as previously described. ${ }^{3}$

Polymer microcavities were grown via spin-coating of different solutions at $7500 \mathrm{rpm}$. A DBR consisting of 25 bilayers was fabricated on a $22 \times 22 \mathrm{~mm}$ glass substrate by alternated depositions of PVK in toluene $(28 \mathrm{~g} / \mathrm{L})$ and CA in 4-hydroxy4-methyl-2-pentanone ( $35 \mathrm{~g} / \mathrm{L})$. The sample was annealed at $80{ }^{\circ} \mathrm{C}$ for $1 \mathrm{~min}$ after each PVK deposition to avoid percolation of CA solvent through the PVK layers. ${ }^{98}$ This technique allows to finely control layer thickness and interfacial roughness to about $1 \mathrm{~nm}$ for commodity polymers. ${ }^{59,99}$ For less processable polymers but with higher dielectric contrast roughness could be less controlled. Notice also that among commodity polymers, CA and PVK are the ones showing the largest dielectric contrast and at the same time guaranteeing optimal mutual processability for multilayers structures. ${ }^{77}$ The DiRs:PS nanocomposite was prepared by dissolving polystyrene into a DiRs:toluene dispersion (40 g/L, PS; $0.8 \mathrm{~g} / \mathrm{L}$, DiRs). This composite solution was spun-cast at $4800 \mathrm{rpm}$ on the previously grown DBR and annealed at $80{ }^{\circ} \mathrm{C}$ for $10 \mathrm{~min}$. Finally, another 25 bilayers of CA and PVK were fabricated on top of the DiRs:PS nanocomposite layer by alternated spin-coating to complete the microcavity. The resulting structure is schematized in Figure 1b. Growing conditions have been adjusted to achieve DBRs in the $\lambda / 4$ condition and to match the cavity mode with the DiRs emission peak (Figure 2a-c). Our all-polymer microcavities can be peeled-off from the substrates they were fabricated on as shown in Figure 1a, where the red fluorescence is clearly observed under violet excitation. Our samples are flexible and can be adapted to complex surfaces. As reference for PL measurements (spectral shape, intensity, quantum yield, and lifetime), we spin-coated a DiRs:PS nanocomposite layer as described above for the microcavity on a glass substrate coated with a CA film to obtain the same thickness.

Reflectance spectra were measured with a system based on an Avantes compact spectrometer (resolution $1.4 \mathrm{~nm}$ ) as 
previously described. ${ }^{64,69,77}$ Angle resolved spectra have been recorded by a homemade setup (angular resolution $\leq 1^{\circ}$ ).

Steady-state PL measurements were performed by exciting the samples with an Oxxius $405 \mathrm{~nm} \mathrm{CW}$ laser focused on a $1 \mathrm{~mm}^{2}$ spot and the fluorescence was collected with an Ocean Optics Jazz COMBO 2 compact modular spectrometers (530-880 nm, $0.5 \mathrm{~nm}$ resolution). Time-resolved PL measurements were recorded using a $405 \mathrm{~nm}$ LDH-P-C-405 laser combined with a PDL $800 \mathrm{~B}$ driver (40 ps pulse width, $5 \mathrm{MHz}$ repetition rate) and a PicoQuant Time Correlated Single Photon Counting system (Time Harp 260 PICO board, 25 ps temporal resolution; PMA Hybrid 40 detector, 140 ps response time) equipped with a Solar Laser Systems monochromator. Each decay has been measured 17 times over different spots on sample surface and, for each measurement, photoluminescence lifetimes have been retrieved using PicoQuant FluoFit Pro fitting software (accounting for the instrument response function). The decay parameters reported in this work are the average over the 17 fits.

External PL quantum efficiency for microcavities and references were measured as described by de Mello et al. ${ }^{100}$ using an integrating sphere (Avantes AvaSphere-50) fibercoupled with the Oxxius $405 \mathrm{~nm}$ laser previously described, and an Avantes AvaSpec-2048 calibrated spectrometer (200-1150 nm, $1.4 \mathrm{~nm}$ resolution).

\section{ASSOCIATED CONTENT}

\section{S Supporting Information}

The Supporting Information is available free of charge on the ACS Publications website at DOI: 10.1021/acsphotonics.7b00330.

DiRs TEM characterization and size distribution. Confocal microscopy of nanocomposite film. Deconvolution of reference PL spectrum and obtained parameters. Extinction coefficient estimation procedure. Angle resolved transmittance spectra of microcavity. Peak normalized PL spectra. Angle resolved microcavity PL spectrum map. Toluene dispersion time-resolved PL decay and fit parameters (PDF).

\section{AUTHOR INFORMATION}

\section{Corresponding Author}

*E-mail: davide.comoretto@unige.it.

\section{ORCID}

Roman Krahne: 0000-0003-0066-7019

Davide Comoretto: 0000-0002-2168-2851

\section{Present Address}

${ }^{\S}$ ICFO Institute of Photonic Sciences, Barcelona, Spain.

\section{Author Contributions}

All authors have contributed to the final version of the manuscript.

\section{Notes}

The authors declare no competing financial interest.

\section{ACKNOWLEDGMENTS}

Work at UNIGE was funded by the Italian Ministry of University, Research and Instruction through the "Progetti di Ricerca di Rilevante Interesse Nazionale 2010-2011” Program (Materiali Polimerici Nanostrutturati con strutture molecolari e cristalline mirate, per tecnologie avanzate e per l'ambiente, 2010XLLNM3). P.L. is thankful for support by the European Union's Horizon 2020 research and innovation program under the Marie Sklodowska-Curie Grant Agreement No. 643238. We thank F. De Donato for providing the DiRs used in this work.

\section{REFERENCES}

(1) Angeloni, I.; Raja, W.; Brescia, R.; Polovitsyn, A.; De Donato, F.; Canepa, M.; Bertoni, G.; Proietti Zaccaria, R.; Moreels, I. Disentangling the Role of Shape, Ligands, and Dielectric Constants in the Absorption Properties of Colloidal CdSe/CdS Nanocrystals. ACS Photonics 2016, 3, 58-67.

(2) Nasilowski, M.; Mahler, B.; Lhuillier, E.; Ithurria, S.; Dubertret, B. Two-Dimensional Colloidal Nanocrystals. Chem. Rev. 2016, 116, 10934-10982.

(3) Carbone, L.; Nobile, C.; De Giorgi, M.; Sala, F. D.; Morello, G.; Pompa, P.; Hytch, M.; Snoeck, E.; Fiore, A.; Franchini, I. R.; Nadasan, M.; Silvestre, A. F.; Chiodo, L.; Kudera, S.; Cingolani, R.; Krahne, R.; Manna, L. Synthesis and Micrometer-Scale Assembly of Colloidal $\mathrm{CdSe} / \mathrm{CdS}$ Nanorods Prepared by a Seeded Growth Approach. Nano Lett. 2007, 7, 2942-2950.

(4) Chen, Y.; Vela, J.; Htoon, H.; Casson, J. L.; Werder, D. J.; Bussian, D. A.; Klimov, V. I.; Hollingsworth, J. A. Giant" Multishell CdSe Nanocrystal Quantum Dots with Suppressed Blinking. J. Am. Chem. Soc. 2008, 130, 5026-5027.

(5) Christodoulou, S.; Vaccaro, G.; Pinchetti, V.; De Donato, F.; Grim, J. Q.; Casu, A.; Genovese, A.; Vicidomini, G.; Diaspro, A.; Brovelli, S.; Manna, L.; Moreels, I. Synthesis of Highly Luminescent Wurtzite CdSe/CdS Giant-shell Nanocrystals Using a Fast Continuous Injection Route. J. Mater. Chem. C 2014, 2, 3439-3447.

(6) Dai, X.; Zhang, Z.; Jin, Y.; Niu, Y.; Cao, H.; Liang, X.; Chen, L.; Wang, J.; Peng, X. Solution-processed, High-performance Lightemitting Diodes Based on Quantum Dots. Nature 2014, 515, 96-99.

(7) Nam, S.; Oh, N.; Zhai, Y.; Shim, M. High Efficiency and Optical Anisotropy in Double-Heterojunction Nanorod Light-Emitting Diodes. ACS Nano 2015, 9, 878-885.

(8) Yang, Y.; Zheng, Y.; Cao, W.; Titov, A.; Hyvonen, J.; Manders, J. R.; Xue, J.; Holloway, P. H.; Qian, L. High-efficiency Light-emitting Devices Based on Quantum Dots with Tailored Nanostructures. Nat. Photonics 2015, 9, 259-266.

(9) Lin, C. H.; Lafalce, E.; Jung, J.; Smith, M. J.; Malak, S. T.; Aryal, S.; Yoon, Y. J.; Zhai, Y.; Lin, Z.; Vardeny, Z. V.; Tsukruk, V. V. Core/ Alloyed-Shell Quantum Dot Robust Solid Films with High Optical Gains. ACS Photonics 2016, 3, 647-658.

(10) Jiang, Y.; Oh, N.; Shim, M. Double-Heterojunction Nanorod Light-Emitting Diodes with High Efficiencies at High Brightness Using Self-Assembled Monolayers. ACS Photonics 2016, 3, 1862-1868.

(11) Grim, J. Q.; Christodoulou, S.; Di Stasio, F.; Krahne, R.; Cingolani, R; Manna, L.; Moreels, I. Continuous-wave Biexciton Lasing at Room Temperature Using Solution-processed Quantum Wells. Nat. Nanotechnol. 2014, 9, 891-895.

(12) Min, B.; Kima, S.; Okamoto, K.; Yang, L.; Scherer, A.; Atwater, H.; Vahalab, K. Ultralow Threshold On-chip Microcavity Nanocrystal Quantum Dot Lasers. Appl. Phys. Lett. 2006, 89, 191124.

(13) Dang, C.; Lee, J.; Breen, C.; Steckel, J. S.; Coe-Sullivan, S.; Nurmikko, A. Red, Green and Blue Lasing Enabled by Single-exciton Gain in Colloidal Quantum Dot Films. Nat. Nanotechnol. 2012, 7, 335-339.

(14) Schaefer, J.; Mondia, J. P.; Sharma, R.; Lu, Z. H.; Susha, A. S.; Rogach, A. L.; Wang, L. J. Quantum Dot Microdrop Laser. Nano Lett. 2008, 8, 1709-1712.

(15) Kelestemur, Y.; Cihan, A. F.; Guzelturk, B.; Demir, H. V. Typetunable Amplified Spontaneous Emission from Core-seeded CdSe/ CdS Nanorods Controlled by Exciton-exciton Interaction. Nanoscale 2014, 6, 8509-8514.

(16) Gollner, C.; Ziegler, J.; Protesescu, L.; Dirin, D. N.; Lechner, R. T.; Fritz-Popovski, G.; Sytnyk, M.; Yakunin, S.; Rotter, S.; Yousefi Amin, A. A.; Vidal, C.; Hrelescu, C.; Klar, T. A.; Kovalenko, M. V.; Heiss, W. Random Lasing with Systematic Threshold Behavior in Films of CdSe/CdS Core/Thick-Shell Colloidal Quantum Dots. ACS Nano 2015, 9, 9792-9801. 
(17) Di Stasio, F.; Polovitsyn, A.; Angeloni, I.; Moreels, I.; Krahne, R. Broadband Amplified Spontaneous Emission and Random Lasing from Wurtzite CdSe/CdS "Giant-Shell” Nanocrystals. ACS Photonics 2016, 3, 2083-2088.

(18) Pu, C.; Peng, X. To Battle Surface Traps on CdSe/CdS Core/ Shell Nanocrystals: Shell Isolation versus Surface Treatment. J. Am. Chem. Soc. 2016, 138, 8134-8142.

(19) Jones, M.; Lo, S. S.; Scholes, G. D. Quantitative Modeling of the Role of Surface Traps in CdSe/CdS/ZnS Nanocrystal Photoluminescence Decay Dynamics. Proc. Natl. Acad. Sci. U. S. A. 2009, 106, 3011-3016.

(20) Jones, M.; Kumar, S.; Lo, S. S.; Scholes, G. D. Exciton Trapping and Recombination in Type II CdSe/CdTe Nanorod Heterostructures. J. Phys. Chem. C 2008, 112, 5423-5431.

(21) Lin, W.; Niu, Y.; Meng, R.; Huang, L.; Cao, H.; Zhang, Z.; Qin, H.; Peng, X. Shell-thickness dependent optical properties of CdSe/ CdS core/shell nanocrystals coated with thiol ligands. Nano Res. 2016, 9, 260-271.

(22) Zhou, R.; Stalder, R.; Xie, D.; Cao, W.; Zheng, Y.; Yang, Y.; Plaisant, M.; Holloway, P. H.; Schanze, K. S.; Reynolds, J. R.; Xue, J. Enhancing the Efficiency of Solution-Processed Polymer:Colloidal Nanocrystal Hybrid Photovoltaic Cells Using Ethanedithiol Treatment. ACS Nano 2013, 7, 4846-4854.

(23) Zavelani-Rossi, M.; Krahne, R.; Della Valle, G.; Longhi, S.; Franchini, I. R.; Girardo, S.; Scotognella, F.; Pisignano, D.; Manna, L.; Lanzani, G.; Tassone, F. Self-assembled CdSe/CdS Nanorod Microlasers Fabricated from Solution by Capillary Jet Deposition. Laser Photonics Rev. 2012, 6, 678-683.

(24) Zavelani-Rossi, M.; Lupo, M. G.; Krahne, R.; Manna, L.; Lanzani, G. Lasing in Self-assembled Microcavities of CdSe/CdS Core/shell Colloidal Quantum Rods. Nanoscale 2010, 2, 931-935.

(25) Moreels, I.; Rainò, G.; Gomes, R.; Hens, Z.; Stöferle, T.; Mahrt, R. F. Nearly Temperature-Independent Threshold for Amplified Spontaneous Emission in Colloidal CdSe/CdS Quantum Dot-in-Rods. Adv. Mater. 2012, 24, OP231-OP235.

(26) Fischer, A.; Rollny, L.; Pan, J.; Carey, G. H.; Thon, S. M.; Hoogland, S.; Voznyy, O.; Zhitomirsky, D.; Kim, J. Y.; Bakr, O. M.; Sargent, E. H. Directly Deposited Quantum Dot Solids Using a Colloidally Stable Nanoparticle Ink. Adv. Mater. 2013, 25, 5742-5749.

(27) Di Stasio, F.; Grim, J. Q.; Lesnyak, V.; Rastogi, P.; Manna, L.; Moreels, I.; Krahne, R. Single-Mode Lasing from Colloidal WaterSoluble CdSe/CdS Quantum Dot-in-Rods. Small 2015, 11, 13281334.

(28) Canazza, G.; Scotognella, F.; Lanzani, G.; De Silvestri, S.; Zavelani-Rossi, M.; Comoretto, D. Lasing from All-polymer Microcavities. Laser Phys. Lett. 2014, 11, 035804.

(29) Goldenberg, L. M.; Lisinetskii, V.; Schrader, S. All-polymer Spin-coated Organic Vertical-cavity Surface-emitting Laser with High Conversion Efficiency. Appl. Phys. B: Lasers Opt. 2015, 120, 271-277.

(30) Organic and Hybrid Photonic Crystals, 1st ed.; Comoretto, D., Ed.; Springer International Publishing: Switzerland, 2015; p 497.

(31) Fogg, D. E.; Radzilowski, L. H.; Dabbousi, B. O.; Schrock, R. R.; Thomas, E. L.; Bawendi, M. G. Fabrication of Quantum Dot-Polymer Composites: Semiconductor Nanoclusters in Dual-Function Polymer Matrices with Electron-Transporting and Cluster-Passivating Properties. Macromolecules 1997, 30, 8433-8439.

(32) Zanella, M.; Gomes, R.; Povia, M.; Giannini, C.; Zhang, Y.; Riskin, A.; Bael, M. V.; Hens, Z.; Manna, L. Self-Assembled Multilayers of Vertically Aligned Semiconductor Nanorods on Device-Scale Areas. Adv. Mater. 2011, 23, 2205-2209.

(33) Arciniegas, M. P.; Stasio, F. D.; Li, H.; Altamura, D.; De Trizio, L.; Prato, M.; Scarpellini, A.; Moreels, I.; Krahne, R.; Manna, L. SelfAssembled Dense Colloidal $\mathrm{Cu}_{2}$ Te Nanodisk Networks in P3HT Thin Films with Enhanced Photocurrent. Adv. Funct. Mater. 2016, 26, $4535-4542$.

(34) Li, G.; Tan, Z.-K.; Di, D.; Lai, M. L.; Jiang, L.; Lim, J. H.-W.; Friend, R. H.; Greenham, N. C. Efficient Light-Emitting Diodes Based on Nanocrystalline Perovskite in a Dielectric Polymer Matrix. Nano Lett. 2015, 15, 2640-2644.
(35) Rippa, M.; Capasso, R.; Mormile, P.; De Nicola, S.; Zanella, M.; Manna, L.; Nenna, G.; Petti, L. Bragg Extraction of Light in 2D Photonic Thue-Morse Quasicrystals Patterned in Active CdSe/CdS Nanorod-polymer Nanocomposites. Nanoscale 2013, 5, 331-336.

(36) Peng, S.; Zhang, R.; Chen, V. H.; Khabiboulline, E. T.; Braun, P.; Atwater, H. A. Three-Dimensional Single Gyroid Photonic Crystals with a Mid-Infrared Bandgap. ACS Photonics 2016, 3, 1131-1137.

(37) Joannopulos, J. D.; Meade, R. D.; Win, J. N. Photonic Crystals: Molding the Flow of the Light; Princeton University Press: Princeton, 1995.

(38) Yablonovitch, E. Inhibited Spontaneous Emission in solid-State Physics and Electronics. Phys. Rev. Lett. 1987, 58, 2059.

(39) Song, H.; Singer, K.; Lott, J.; Wu, Y.; Zhou, J.; Andrews, J.; Baer, E.; Hiltner, A.; Weder, C. Continuous Melt Processing of All-polymer Distributed Feedback Lasers. J. Mater. Chem. 2009, 19, 7520-7524.

(40) Toray Picasus Product Presentation. http://www.toray.com/ products/films/fil 012.html (accessed 2017/03/01).

(41) Hou, L.; Hou, Q.; Mo, Y.; Peng, J.; Cao, Y. All-organic flexible polymer microcavity light-emitting diodes using $3 \mathrm{M}$ reflective multilayer polymer mirrors. Appl. Phys. Lett. 2005, 87, 243504.

(42) Weber, M. F.; Stover, C. A.; Gilbert, L. R.; Nevitt, T. J.; Ouderkirk, A. J. Giant Birefringent Optics in Multilayer Polymer Mirrors. Science 2000, 287, 2451-2456.

(43) Kim, J. C.; Cakmak, M.; Zhou, X. Effect of Composition on Orientation, Optical and Mechanical Properties of Bi-axially Drawn PEN and PEN/PEI Blend Films. Polymer 1998, 39, 4225-4234.

(44) Ponting, M.; Hiltner, A.; Baer, E. Polymer Nanostructures by Forced Assembly: Process, Structure, and Properties. Macromol. Symp. 2010, 294, 19-32.

(45) Lee, J.-H.; Koh, C. Y.; Singer, J. P.; Jeon, S.-J.; Maldovan, M.; Stein, O.; Thomas, E. L. 25th Anniversary Article: Ordered Polymer Structures for the Engineering of Photons and Phonons. Adv. Mater. 2014, 26, 532-569.

(46) Russo, M.; Campoy-Quiles, M.; Lacharmoise, P.; Ferenczi, T. A. M.; Garriga, M.; Caseri, W. R.; Stingelin, N. One-pot Synthesis of Polymer/inorganic Hybrids: Toward Readily Accessible, Low-loss, and Highly Tunable Refractive Index Materials and Patterns. J. Polym. Sci., Part B: Polym. Phys. 2012, 50, 65-74.

(47) Park, T. J.; Hwang, S. K.; Park, S.; Cho, S. H.; Park, T. H.; Jeong, B.; Kang, H. S.; Ryu, D. Y.; Huh, J.; Thomas, E. L.; Park, C. Electrically Tunable Soft-Solid Block Copolymer Structural Color. ACS Nano 2015, 9, 12158-12167.

(48) Chiang, Y.-W.; Chou, C.-Y.; Wu, C.-S.; Lin, E.-L.; Yoon, J.; Thomas, E. L. Large-Area Block Copolymer Photonic Gel Films with Solvent-Evaporation-Induced Red- and Blue-Shift Reflective Bands. Macromolecules 2015, 48, 4004-4011.

(49) Calvo, M. E.; Colodrero, S.; Hidalgo, N.; Lozano, G.; LopezLopez, C.; Sanchez-Sobrado, O.; Miguez, H. Porous one dimensional photonic crystals: novel multifunctional materials for environmental and energy applications. Energy Environ. Sci. 2011, 4, 4800-4812.

(50) Calvo, M. E.; Colodrero, S.; Rojas, T. C.; Anta, J. A.; Ocaña, M.; Míguez, H. Photoconducting Bragg Mirrors based on $\mathrm{TiO}_{2}$ Nanoparticle Multilayers. Adv. Funct. Mater. 2008, 18, 2708-2715.

(51) Puzzo, D. P.; Scotognella, F.; Zavelani-Rossi, M.; Sebastian, M.; Lough, A. J.; Manners, I.; Lanzani, G.; Tubino, R.; Ozin, G. A. Distributed Feedback Lasing from a Composite Poly(phenylene vinylene)-Nanoparticle One-Dimensional Photonic Crystal. Nano Lett. 2009, 9, 4273-4278.

(52) Lotsch, B. V.; Ozin, G. A. Photonic Clays: A New Family of Functional 1D Photonic Crystals. ACS Nano 2008, 2, 2065-2074.

(53) Jiménez-Solano, A.; Galisteo-López, J. F.; Míguez, H. Fine Tuning the Emission Properties of Nanoemitters in Multilayered Structures by Deterministic Control of their Local Photonic Environment. Small 2015, 11, 2727-2732.

(54) Calvo, M. E.; Castro Smirnov, J. R.; Míguez, H. Novel approaches to flexible visible transparent hybrid films for ultraviolet protection. J. Polym. Sci., Part B: Polym. Phys. 2012, 50, 945-956. 
(55) Fink, Y.; Winn, J. N.; Fan, S.; Chen, C.; Michel, J.; Joannopoulos, J. D.; Thomas, E. L. A Dielectric Omnidirectional Reflector. Science 1998, 282, 1679-1682.

(56) Menon, V. M.; Luberto, M.; Valappil, N. V.; Chatterjee, S. Lasing from In GaP quantum dots in a spin-coated flexible microcavity. Opt. Express 2008, 16, 19535-19540.

(57) Valappil, N. V.; Luberto, M.; Menon, V. M.; Zeylikovich, I.; Gayen, T. K.; Franco, J.; Das, B. B.; Alfano, R. R. Solution processed microcavity structures with embedded quantum dots. Photonics Nanostruct. Fundam. Appl. 2007, 5, 184-188.

(58) Goldenberg, L. M.; Lisinetskii, V.; Schrader, S. Fast and simple fabrication of organic Bragg mirrors - application to plastic microchip lasers. Laser Phys. Lett. 2013, 10, 055808.

(59) Radice, S. V.; Srinivasan, P.; Comoretto, D.; Gazzo, S. Onedimensional planar photonic crystals including fluoropolymer compositions and corresponding fabrication methods, WO 2016/ 087439 Al, 2016.

(60) Najda, S. P. An optoelectronic semiconductor device, EP0795941 A1, 1997.

(61) Ryan, H.; Maria, C. Photonic Polymer Multilayers for Colorimetric Radiation Sensing, US2016/0252625 A1, 2016.

(62) Chang, J.-H. Method for manufacturing a reflective optical film, US2016/0245971 A1, 2016.

(63) Bai, Z.; Joo, J.; Sung, I.-K.; Taylor, J. C. Multilayer Polymer Composite for encapsulating quantum dots, US2016/0272885 A1, 2016.

(64) Lova, P.; Manfredi, G.; Boarino, L.; Comite, A.; Laus, M.; Patrini, M.; Marabelli, F.; Soci, C.; Comoretto, D. Polymer Distributed Bragg Reflectors for Vapor Sensing. ACS Photonics 2015, 2, 537-543.

(65) Goldenberg, L. M.; Lisinetskii, V.; Gritsai, Y.; Stumpe, J.; Schrader, S. Second order DFB lasing using reusable grating inscribed in azobenzene-containing material. Opt. Mater. Express 2012, 2, 1119.

(66) Lova, P.; Bastianini, C.; Giusto, P.; Patrini, M.; Rizzo, P.; Guerra, G.; Iodice, M.; Soci, C.; Comoretto, D. Label-Free Vapor Selectivity in Poly(p-Phenylene Oxide) Photonic Crystal Sensors. ACS Appl. Mater. Interfaces 2016, 8, 31941-31950.

(67) Lova, P.; Manfredi, G.; Boarino, L.; Laus, M.; Urbinati, G.; Losco, T.; Marabelli, F.; Caratto, V.; Ferretti, M.; Castellano, M.; Soci, C.; Comoretto, D. Hybrid $\mathrm{ZnO}$ :polystyrene nanocomposite for allpolymer photonic crystals. Phys. Stat. Solidi C 2015, 12, 158-162.

(68) Komikado, T.; Yoshida, S.; Umegaki, S. Surface-emitting distributed-feedback dye laser of a polymeric multilayer fabricated by spin coating. Appl. Phys. Lett. 2006, 89, 061123.

(69) Knarr, R. J., III; Manfredi, G.; Martinelli, E.; Pannocchia, M.; Repetto, D.; Mennucci, C.; Solano, I.; Canepa, M.; Buatier de Mongeot, F.; Galli, G.; Comoretto, D. In-plane anisotropic photoresponse in all-polymer planar microcavities. Polymer 2016, 84, 383390.

(70) Moritsugu, M.; Kim, S.-n.; Kubo, S.; Ogata, T.; Nonaka, T.; Sato, O.; Kurihara, S. Photoswitching properties of photonic crystals infiltrated with polymer liquid crystals having azobenzene side chain groups with different methylene spacers. React. Funct. Polym. 2011, 71, $30-35$.

(71) Moritsugu, M.; Ishikawa, T.; Kawata, T.; Ogata, T.; Kuwahara, Y.; Kurihara, S. Thermal and Photochemical Control of Molecular Orientation of Azo-Functionalized Polymer Liquid Crystals and Application for Photo-Rewritable Paper. Macromol. Rapid Commun. 2011, 32, 1546-1550.

(72) Fornasari, L.; Floris, F.; Patrini, M.; Comoretto, D.; Marabelli, F. Demonstration of fluorescence enhancement via Bloch surface waves in all-polymer multilayer structures. Phys. Chem. Chem. Phys. 2016, 18, 14086-14093.

(73) Comoretto, D.; Dellepiane, G.; Cuniberti, C.; Rossi, L.; Borghesi, A.; LeMoigne, J. Photoinduced Absorption of Oriented Poly[1,6-di(N-carbazolyl)-2,4-hexadiyne]. Phys. Rev. B: Condens. Matter Mater. Phys. 1996, 53, 15653-15659.

(74) Comoretto, D.; Moggio, I.; Cuniberti, C.; Dellepiane, G.; Giardini, M. E.; Borghesi, A. Long-lived photoexcited states in polydiacetylenes with different molecular and supramolecular organization. Phys. Rev. B: Condens. Matter Mater. Phys. 1997, 56, 10264-10270.

(75) Moroni, L.; Salvi, P. R.; Gellini, C.; Dellepiane, G.; Comoretto, D.; Cuniberti, C. Two-photon spectroscopy of pi-conjugated polymers: The case of poly[1,6-bis(3,6-dihexadecyl-N-carbazolyl)2,4-hexadiyne] (polyDCHD-HS). J. Phys. Chem. A 2001, 105, $7759-7764$.

(76) Skorobogatiy, M.; Yang, J. Fundamentals of Photonic Crystal Guiding; Cambridge University Press, 2009.

(77) Gazzo, S.; Manfredi, G.; Poetzsch, R.; Wei, Q.; Alloisio, M.; Voit, B.; Comoretto, D. High refractive index hyperbranched polyvinylsulfides for planar one-dimensional all-polymer photonic crystals. J. Polym. Sci., Part B: Polym. Phys. 2016, 54, 73-80.

(78) Manfredi, G.; Mayrhofer, C.; Kothleitner, G.; Schennach, R.; Comoretto, D. Cellulose ternary photonic crystal created by solution processing. Cellulose 2016, 23, 2853-2862.

(79) Saleh, B. E. A.; Teich, M. C. Fundamentals of Photonics; Wiley, 2007.

(80) Frezza, L.; Patrini, M.; Liscidini, M.; Comoretto, D. Directional Enhancement of Spontaneous Emission in Polymer Flexible Microcavities. J. Phys. Chem. C 2011, 115, 19939-19946.

(81) Barth, M.; Gruber, A.; Cichos, F. Spectral and Angular Redistribution of Photoluminescence Near a Photonic Stop Band. Phys. Rev. B: Condens. Matter Mater. Phys. 2005, 72, 085129.

(82) Björk, G.; Machida, S.; Yamamoto, Y.; Igeta, K. Modification of spontaneous emission rate in planar dielectric microcavity structures. Phys. Rev. A: At., Mol., Opt. Phys. 1991, 44, 669-681.

(83) Björk, G. On the spontaneous lifetime change in an ideal planar microcavity - transition from a mode continuuum to quantized modes. IEEE J. Quantum Electron. 1994, 30, 2314-2318.

(84) Schubert, E. F.; Hunt, N. E. J.; Micovic, M.; Malik, R. J.; Sivco, D. L.; Cho, A. Y.; Zydzik, G. J. Highly Efficient Light-Emitting Diodes with Microcavities. Science 1994, 265, 943-945.

(85) Liscidini, M.; Andreani, L. C. Photonic Crystals: An Introductory Survey. In Organic and Hybrid Photonic Crystals; Comoretto, D., Ed.; Springer International Publishing: Switzerland, 2015; pp 3-29.

(86) Berti, L.; Cucini, M.; Di Stasio, F.; Comoretto, D.; Galli, M.; Marabelli, F.; Manfredi, N.; Marinzi, C.; Abbotto, A. Spectroscopic Investigation of Artificial Opals Infiltrated with a Heteroaromatic Quadrupolar Dye. J. Phys. Chem. C 2010, 114, 2403-2413.

(87) Rabouw, F. T.; Lunnemann, P.; van Dijk-Moes, R. J. A.; Frimmer, M.; Pietra, F.; Koenderink, A. F.; Vanmaekelbergh, D. Reduced Auger Recombination in Single CdSe/CdS Nanorods by One-Dimensional Electron Delocalization. Nano Lett. 2013, 13, 48844892.

(88) Galland, C.; Ghosh, Y.; Steinbruck, A.; Sykora, M.; Hollingsworth, J. A.; Klimov, V. I.; Htoon, H. Two types of luminescence blinking revealed by spectroelectrochemistry of single quantum dots. Nature 2011, 479, 203-207.

(89) Mallek-Zouari, I.; Buil, S.; Quélin, X.; Mahler, B.; Dubertret, B.; Hermier, J.-P. Plasmon assisted single photon emission of CdSe/CdS nanocrystals deposited on random gold film. Appl. Phys. Lett. 2010, 97, 053109.

(90) Schlegel, G.; Bohnenberger, J.; Potapova, I.; Mews, A. Fluorescence Decay Time of Single Semiconductor Nanocrystals. Phys. Rev. Lett. 2002, 88, 137401.

(91) Califano, M. Photoinduced Surface Trapping and the Observed Carrier Multiplication Yields in Static CdSe Nanocrystal Samples. ACS Nano 2011, 5, 3614-3621.

(92) Kaur, G.; Kaur, H.; Tripathi, S. K. Fluorescence Relaxation Dynamics of CdSe and CdSe/CdS Core/shell Quantum Dots. AIP Conf. Proc. 2013, 1591, 420-422.

(93) Jones, M.; Lo, S. S.; Scholes, G. D. Quantitative modeling of the role of surface traps in $\mathrm{CdSe} / \mathrm{CdS} / \mathrm{ZnS}$ nanocrystal photoluminescence decay dynamics. Proc. Natl. Acad. Sci. U. S. A. 2009, 106, 30113016. 
(94) Gérard, J.-M.; Gayral, B. Strong Purcell Effect for InAs Quantum Boxes in Three-Dimensional Solid-State Microcavities. J. Lightwave Technol. 1999, 17, 2089-2095.

(95) Baba, T.; Sano, D. Low-Threshold Lasing and Purcell Effect in Microdisk Lasers at Room Temperature. IEEE J. Sel. Top. Quantum Electron. 2003, 9, 1340-1346.

(96) Alloisio, M.; Rusu, M.; Ottonello, S.; Ottonelli, M.; Thea, S.; Comoretto, D. Synthesis of Fluorescent Core-Shell Metal Nanohybrids: A Versatile Approach. Materials 2016, 9, 997.

(97) Gehr, R. J.; Boyd, R. W. Optical Properties of Nanostructured Optical Materials. Chem. Mater. 1996, 8, 1807-1819.

(98) Komikado, T.; Inoue, A.; Masuda, K.; Ando, T.; Umegaki, S. Multi-layered mirrors fabricated by spin-coating organic polymers. Thin Solid Films 2007, 515, 3887-3892.

(99) Unger, K.; Resel, R.; Czibula, C.; Ganser, C.; Teichert, C.; Jakopic, G.; Canazza, G.; Gazzo, S.; Comoretto, D. Distributed Bragg reflectors: Morphology of cellulose acetate and polystyrene multila. 16th International Conference on Transparent Optical Networks (ICTON 2014), Graz, Austria, 2014; pp 1-4.

(100) Greenham, N. C.; Samuel, I. D. W.; Hayes, G. R.; Phillips, R. T.; Kessener, Y. A. R. R.; Moratti, S. C.; Holmes, A. B.; Friend, R. H. Measurement of absolute photoluminescence quantum efficiencies in conjugated polymers. Chem. Phys. Lett. 1995, 241, 89-96. 